\title{
ANIMA: Non-conventional Brain-Computer Interfaces in Robot Control through Electroencephalography and Electrooculography, ARP Module
}

\author{
Luis F. Reina, Gerardo Martínez, Mario Valdeavellano, Marie Destarac, \\ and Carlos Esquit
}

Department of Electronics Engineering, Del Valle de Guatemala University

\begin{abstract}
ANIMA has as a primary objective to compare three nonconventional human com-puter interfaces that comply with the industrial robot ST Robotics R-17 instructions. This mod-ule, Alpha Waves Related Potentials ARP- explains how brain waves are obtained, processed, analyzed and identified depending on their frequency. This module makes use of the Open EEG Project's open hardware monitor for brain wave activity, called the modular EEG. The brain waves are obtained through an electrode cap complying with the international 10-20 system for electrode positioning. The brain waves are processed with a fast Fourier transform using a mi-crocontroller and analyzed in software identifying the alpha wave's contribution. A program identifies the amount of time that alpha wave generation was maintained through concentration, and instructions are sent to the robotic arm, executing one of four pre-defined routines. Thirty percent of the users attained control over the robotic arm with the human computer interface.
\end{abstract}

\section{Introduction}

Brain waves can be obtained through electroencephalography -EEG- using an electrode cap. The electrodes obtain the brain's electrical variations caused by the neuronal interaction. To be able to observe potentials (micro volts scale) from a specific region of the brain, a comparison between two electrodes is needed; one at an area of reference and the other at the area of interest. The international system 10-20 for electrode positioning was created to standardize the position of electrodes in areas of interest.

Brain waves are classified according to their frequency; delta $(0.2-3.5 \mathrm{~Hz})$, theta $(3.5-7.5 \mathrm{~Hz})$, alpha $(7.5-13 \mathrm{~Hz})$, beta $(13-28 \mathrm{~Hz})$ and gamma $(28-40 \mathrm{~Hz})$ [1]. Beta can be divided in two regions: Beta 1 or beta Low $(13-20.5 \mathrm{~Hz})$ and beta 2 or beta High (20.5 a $28 \mathrm{~Hz})$.

Alfa waves are produced in moments of relaxation and tranquility. Most people can produce them when they close their eyes and relax. However, maintaining the generation of alpha waves with eyes wide open is not an easy task [1].

Alpha waves are one type of brain waves detected by electroencephalography EEG- and predominantly originate from the occipital lobe or parietal lobe during 
wakeful relaxation with closed eyes. Alpha waves are reduced with open eyes and drowsiness and sleep.

The graphic representation of the Fast Fourier Transform -FFT- of a wave is a diagram named Fourier spectrum in which the frequency and magnitude of each sinusoid component is represented [2].

ANIMA project has three different modules: the ocular module, motor module and the alpha wave related potentials module -ARP-. All modules were financed by CONCYT (National Council for Science and Technology, for its Spanish abbreviation). Each module makes its own implementation for moving the robotic arm R17; signals produced by the changes in the electric field of the eyes caused by their movement [3], brain waves related to motor tasks [4] and brain waves related to alpha activity. Comparison between the three modules could be done in terms of effectiveness and speed for controlling a Robotics R17 robotic arm. Research in Guatemala is starting to flourish in this area and projects like this one are the very first steps.

\section{Experimental Design}

Brain waves on the parietal lobe are amplified, filtered, sampled and digitalized using the open hardware of the "Open EEG Project" [5] for brain wave acquisition. Brain waves were analyzed by applying the FFT to identify the frequencies involved with concentration. The signals are analyzed using a Python [6] software application to identify the alpha waves produced by concentration. This software identifies the contribution of the theta, alpha, beta1 and beta2 to the frequency spectrum on the FFT. The application uses the alpha waves contribution to identify alpha waves concentration.

Finally, the same application sends instructions to the robotic arm according to the amount of time the user maintained the alpha concentration, thus, executing one out of the four predefined routines for the R17.

\section{Methodology}

Fig. 1 shows the four main steps for this paper. These four steps will be explained on the following sections.

\subsection{Signals Acquisition}

An electrode cap complying with the IS 10-20 was used to obtain the brain waves. Electrodes used were P3 and GND as the reference. A modular EEG was built which has two printed circuit boards -PCBs-, one analog for amplification and filtering, and one digital for communication with a PC. The design for both PCBs is available on the Open EEG Project webpage. The modular EEG PCBs were manufactured at "Universidad del Valle de Guatemala"-UVG- (Fig. 2) and bought in Olimex at the same time [7]. PCBs from both manufacturers were compared and the same results were obtained. An application was made using Python to calibrate the gain obtained with the PCBs. 


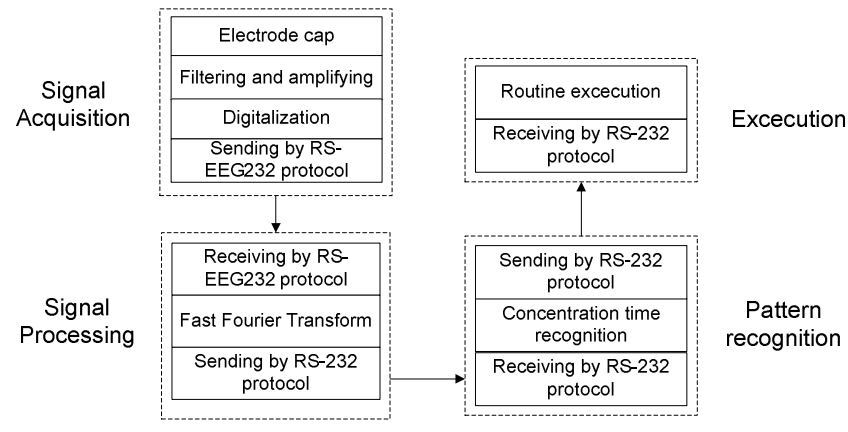

Fig. 1. Steps for the ARP module

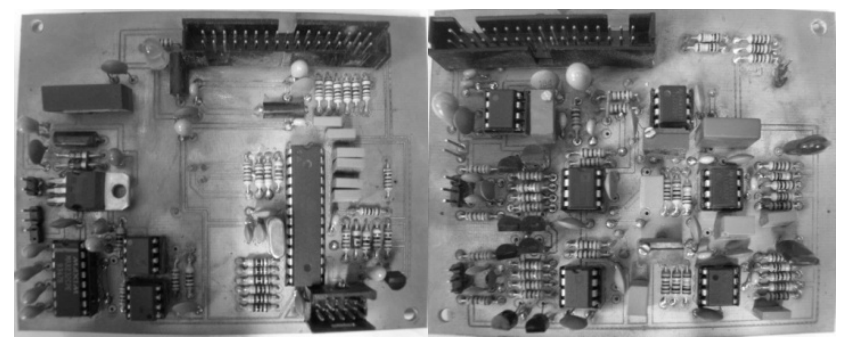

Fig. 2. Digital and analog PCB for Open EEG Projects modular EEG

\subsection{Signal Processing}

The FFT was calculated in a dsPIC microcontroller obtaining data from the modular EEG (AVR microcontroller) communicating through the RS-232EEG protocol and sending the results through RS-232 protocol to a PC (Fig. 3).

A dsPIC microcontroller with two RS-232 (UART) communication ports was used with a $10 \mathrm{MHz} 8 \mathrm{xPLL}$, therefore the dsPIC could work at $80 \mathrm{MHz}$. This allows the dsPIC to receive data from the modular EEG at 57600 baud and send new data to the PC at 115200 baud. This speed is needed so the FFT can be calculated and the data can be sent to a PC in real time. A 256-byte array is sent containing information about the frequency spectrum (magnitude for the $0-128 \mathrm{~Hz}$ range) with $1 \mathrm{~Hz}$ resolution.

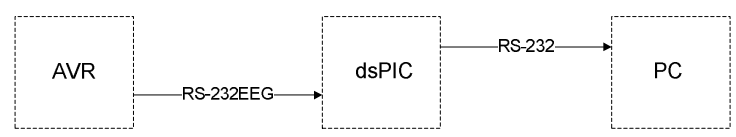

Fig. 3. Communication between the Modular EEG boards and PC

\subsection{Pattern Recognition}

An application was developed using Python to receive the dsPIC data over RS-232 protocol. The application discards the data related to frequencies above $30 \mathrm{~Hz}$. The 
remaining region was divided into frequency regions according to brain waves classification: theta, alpha, beta1 and beta2. For each region an integral of the spectrum was computed. From these integrals the normalized contribution percentage was calculated.

On the GUI (Graphic User Interface) a graph presents the normalized contribution percentage for the four frequency bands (Fig. 4).

Using this application the brain waves are processed comparing the P3 and GND electrode positions from the IS 10-20 cap to identify concentration periods. For a period to be valid there are two requirements: the normalized contribution percentage for alpha waves must be above a threshold of 40 percent (0.4), and a difference of normalized contribution percentage of at least 20 percent $(0.2)$ between alpha waves and the other brain waves (theta, beta1 and beta2) must be obtained. The application identifies concentration periods of $4,6,8$ and 10 seconds.

The application generates different sounds (beeps) for each identified period. The sound acts as a feedback to the user because it is easier to achieve alpha state with closed eyes.

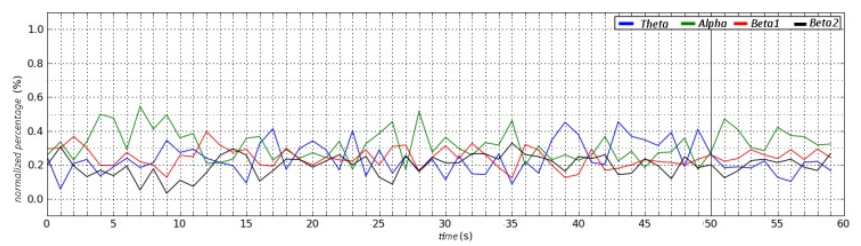

Fig. 4. Normalized percentage shown on the Python GUI

\subsection{Execution}

The GUI described in the previous section identifies alpha concentration periods of time. Another application was developed for the GUI to communicate with the robotic arm R17. This application has two goals: to send an order on each identified period and translate that order to the R17 programmed routines.

Each alpha state period of time was mapped to a R17 routine (Table 1.). The application contains defensive programming to prevent the R17 from going off its limits of movement.

Table 1. Called routine for each alpha state period

\begin{tabular}{cl}
\hline \hline Seconds & \multicolumn{1}{c}{ Routine } \\
\hline 4 & Forward \\
6 & Backward \\
8 & Left \\
10 & Right \\
\hline \hline
\end{tabular}

\section{Results}

Fig. 5 shows an image where the difference in baud rate between the ATMEGA and dsPIC communication can be seen. Also the time of synchronization with the 


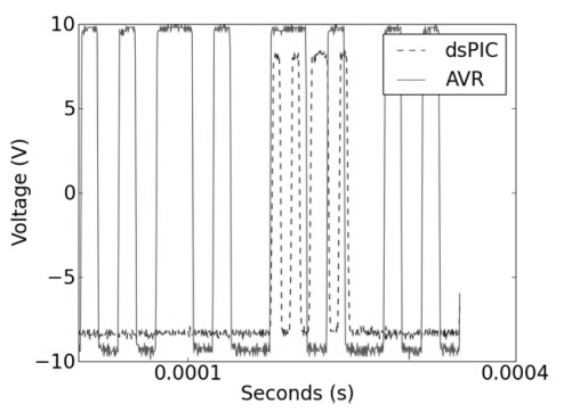

Fig. 5. Delay and difference on baud rate for communication between AVR y dsPIC

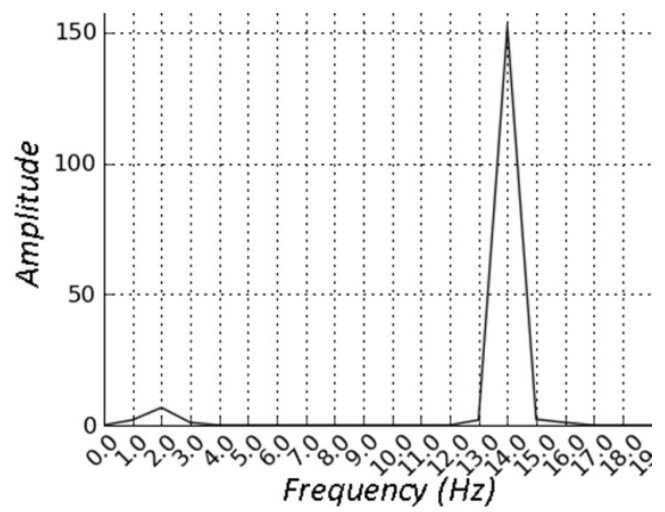

Fig. 6. FFT for a $14 \mathrm{~Hz}$ square wave signal

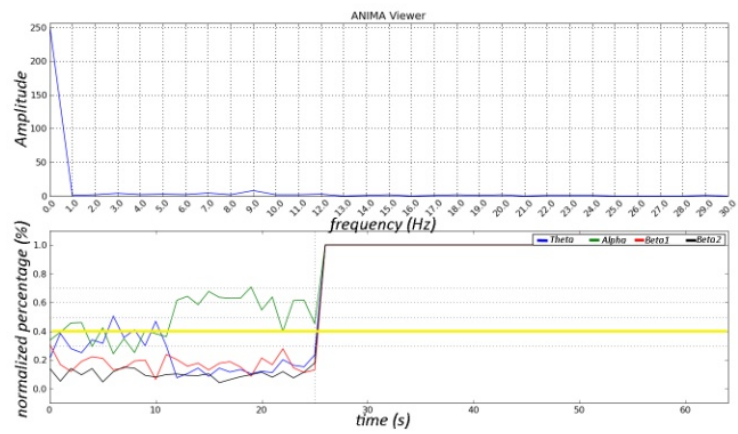

Fig. 7. Python application GUI with real time FFT

RS-232EEG can be seen. The real time FFT is shown in Fig. 6 using a 14Hz square wave signal as input.

The final application GUI is shown in Fig. 7, in which the real time FFT is integrated. In this figure it can be seen that the two required conditions are reached 
after 10 seconds. The yellow line stands for the threshold of alpha waves (green color), and the separation can be easily seen too. The times set for each task are based on various preliminary tests to assure that the alpha waves are being generated voluntarily and are not small involuntary periods of alpha wave generation. After generating alpha waves for a particular task the subject needs to generate 8 seconds of cumulative non-alpha waves to confirm the task, then the subject can start a new period of alpha wave generation and choose a different task.

Tests were made on twenty seven subjects, nine females and eighteen males (Table 2). Testing subjects were asked to close their eyes and then start counting from 1 to 10 until they listened to the first beep. If they achieved this, the second test was to achieve the fourth beep, else the test was ended. Subjects who achieved the fourth beep were able to successfully control all the tasks. In some cases the subject was not able to control the fourth beep (Task 4) but was able to control the other three tasks (Table 5). The order of appearance in Table 5 follows the order of the tests trial.

Table 2. ARP control test of success

\begin{tabular}{lcc}
\hline \hline & Yes & No \\
\hline All & $29.63 \%$ & $70.37 \%$ \\
Men & $38.89 \%$ & $61.11 \%$ \\
Women & $11.11 \%$ & $88.89 \%$ \\
\hline \hline
\end{tabular}

Table 3. Tasks test of success

\begin{tabular}{lcccc}
\hline \hline & Task 1 & Task 2 & Task 3 & Task 4 \\
\hline All & $55.56 \%$ & $33.33 \%$ & $33.33 \%$ & $29.63 \%$ \\
Men & $61.11 \%$ & $38.89 \%$ & $38.89 \%$ & $38.89 \%$ \\
Women & $44.44 \%$ & $22.22 \%$ & $22.22 \%$ & $11.11 \%$ \\
\hline \hline
\end{tabular}

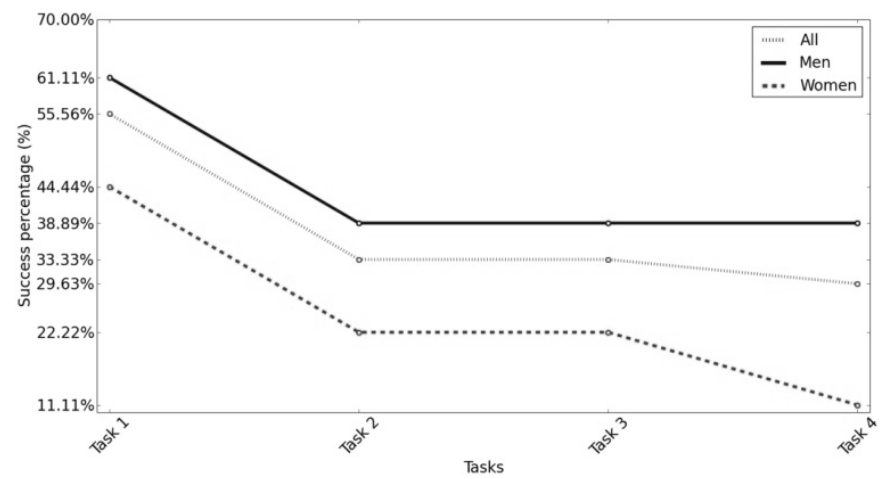

Fig. 8. Success percentage in task tests for women, men and all subjects 
In Table 2 the success in the test is defined as the subject who can control the four tasks, the rest was considered as a failure.

In Table 3 the success for each task is shown. Males show equal difficulty in achieving the tasks two, three and four; either they achieved full control or just achieved to control the first task. It's interesting that women display a much bigger trouble achieving the fourth task. Also the percentage of women who accomplished task 1 is lower. This can be visually verified in Fig. 8.

Table 4 shows the improvement showed on the subjects that didn't achieve all four tasks. Improvement is successful if they managed to accomplish at least one task, in case they started without managing any. Women and men have similar percentage of

Table 4. Unsuccessful subjects that showed improvement

\begin{tabular}{ccc}
\hline \hline & Yes & No \\
\hline All & $36.84 \%$ & $63.16 \%$ \\
Men & $36.36 \%$ & $63.64 \%$ \\
$\begin{array}{c}\text { Wo } \\
\text { men }\end{array}$ & $37.50 \%$ & $62.50 \%$ \\
\hline \hline
\end{tabular}

Table 5. ARP control test results

\begin{tabular}{|c|c|c|c|c|c|c|}
\hline Subject & T1 & T2 & T3 & T4 & Woman & Man \\
\hline Subject 01 & YES & YES & YES & YES & & $\mathrm{X}$ \\
\hline Subject 02 & NO & $\mathrm{NO}$ & NO & NO & $\mathrm{x}$ & \\
\hline Subject 03 & NO & NO & NO & NO & $\mathrm{x}$ & \\
\hline Subject 04 & NO & NO & NO & $\mathrm{NO}$ & & $\mathrm{X}$ \\
\hline Subject 05 & YES & $\mathrm{NO}$ & $\mathrm{NO}$ & $\mathrm{NO}$ & & $\mathrm{X}$ \\
\hline Subject 06 & YES & YES & YES & YES & & $\mathrm{X}$ \\
\hline Subject 07 & YES & YES & YES & YES & & $\mathrm{X}$ \\
\hline Subject 08 & NO & $\mathrm{NO}$ & NO & $\mathrm{NO}$ & & $\mathrm{X}$ \\
\hline Subject 09 & YES & YES & YES & YES & & $\mathrm{X}$ \\
\hline Subject 10 & YES & $\mathrm{NO}$ & NO & $\mathrm{NO}$ & $\mathrm{x}$ & \\
\hline Subject 11 & YES & YES & YES & NO & $\mathrm{x}$ & \\
\hline Subject 12 & YES & YES & YES & YES & & $\mathrm{X}$ \\
\hline Subject 13 & NO & NO & NO & NO & $\mathrm{x}$ & \\
\hline Subject 14 & NO & $\mathrm{NO}$ & NO & $\mathrm{NO}$ & $\mathrm{x}$ & \\
\hline Subject 15 & NO & NO & NO & NO & & $\mathrm{X}$ \\
\hline Subject 16 & NO & $\mathrm{NO}$ & NO & $\mathrm{NO}$ & & $\mathrm{X}$ \\
\hline Subject 17 & YES & NO & NO & $\mathrm{NO}$ & & $\mathrm{X}$ \\
\hline Subject 18 & NO & NO & NO & $\mathrm{NO}$ & & $X$ \\
\hline Subject 19 & YES & YES & YES & YES & & $\mathrm{X}$ \\
\hline Subject 20 & YES & NO & NO & NO & & $\mathrm{X}$ \\
\hline Subject 21 & NO & NO & NO & NO & $\mathrm{x}$ & \\
\hline Subject 22 & YES & NO & NO & NO & $\mathrm{x}$ & \\
\hline Subject 23 & NO & $\mathrm{NO}$ & NO & $\mathrm{NO}$ & & $\mathrm{X}$ \\
\hline Subject 24 & YES & NO & NO & $\mathrm{NO}$ & & $\mathrm{X}$ \\
\hline Subject 25 & YES & YES & YES & YES & & $\mathrm{X}$ \\
\hline Subject 26 & YES & YES & YES & YES & $\mathrm{x}$ & \\
\hline \multirow[t]{2}{*}{ Subject 27} & $\mathrm{NO}$ & $\mathrm{NO}$ & $\mathrm{NO}$ & $\mathrm{NO}$ & & $\mathrm{X}$ \\
\hline & & & & & $\overline{99}$ & 18 \\
\hline
\end{tabular}

*T $=$ Task 
improvement, around 37 percent, which suggests that improvement doesn't depend on the skill to sustain alpha wave generation. This also suggests that subjects could manage the ARP control with proper training [8], but further studies are required to verify these hypotheses. It's interesting to notice that based on some additional tests, it was observed that alpha waves are affected by group pressure, or any distraction, e.g. music, people talking, or strong noises.

\section{Conclusion}

The hardware and software proposed implements a BCI to control the robotic arm R17 using alpha waves concentration by means of open EEG hardware and software developed at Universidad del Valle de Guatemala.

Thirty percent of the users were able to control the robotic arm through the ARP module. Thirty seven percent of the testing subjects showed an improvement on the BCI control.

The system is to be used as a base for a much complex BCI, as for now it represents the beginning of a non-conventional method of robot control that can be applied to different fields of study. The industrial robotic arm was just used to test the BCI, but it could be applied to any other machine or interface that complies with four basic movements.

The purpose of the entire application is to offer a neuro-feedback system that could be used to help handicapped people, but its actual state is still too basic.

\section{Future Work}

The project could use neuronal networks to recognize patterns for specific activities such as spacial and math-related activities, nevertheless results are not guaranteed.

The developed Python application can be used to make an exhaustive analysis of the brain waves. Data such as hemisphere asymmetry, normalized spectral contribution on each hemisphere, peak frequency and mean spectral area could be used as features. These data can be applied to a selection algorithm to identify which ones can be used for identifying a pattern. Then, a Bayesian Network classifier can be used to distinguish between each task [9] [10].

The use of gamma brain waves to determine high-stress states can be applied to avoid sending commands to the robotic arm and wait until the subject is back to the relaxed state so that commands can continue to be sent.

Some patterns could be found among non-standardized parameters using energy measurements of the spectrum (alpha, beta, theta, delta and gamma).

Mathematical tools like PCA, ICA, wavelets and multivariable statistical signal processing should be used to improve the processing algorithm.

\section{Acknowledgment}

This work was partially supported by the Guatemalan National Council for Science and Technology - CONCYT- (for its Spanish abbreviation) and by the Department of Electronics Engineering at Universidad Del Valle de Guatemala. 


\section{References}

1. Johnston, W.: Silent Music: The Science of Meditation, p. 190. Fordham University Press, [trad.] Carmen Bustos (1997)

2. Transformada de Fourier: Escuela Universitaria de Ingeniería Técnica de Telecomunicación, Universidad Politécnica de Madrid (2009),

http: / / www.diac.upm.es/acceso_profesores/asignaturas/

tdi/tdi/transformadas/pdf/fourier1.pdf

3. Valdeavellano, M.R.: ANIMA: Métodos no convencionales de interfaz en el control de robots a través de la electroencefalografía y la electrooculografía: Módulo ocular. Universidad del Valle de Guatemala, Guatemala (2009)

4. Martínez, G.E.: ANIMA: Métodos no convencionales de interfaz en el control de robots a través de la electroencefalografía y la electrooculografía: Módulo motriz. Universidad del Valle de Guatemala, Guatemala (2009)

5. ModularEEG. OpenEEG Project (2008), http: / / openeeg . sourceforge. net/

6. Python Software Foundation. Python Programming Language (2010), http: / / python.org/

7. Open EEG Gadgets. OLIMEX Ltd. (2009), http://www.olimex.com/gadgets/index.html

8. Palke, A.: Brainathlon: Enhancing Brainwave Control Through Brain-Controlled Game Play, p. 37 (2003), http://www. webkitchen.com/brainathlon/files/thesis.pdf

9. Lee, J.C., Tan, D.S.: Using a low-cost electroencephalograph for task classification in HCI research. In: Symposium on User Interface Software and Technology on Sensing from head to toe, pp. 81-90 (2006)

10. Keirn, Z.A., Aunon, J.I.: A new mode of communication between man and his surroundings. IEEE Transactions on Biomedical Engineering 37(12), 1209-1214 (1990) 